\title{
EuMIXFOR empirical forest mensuration and ring width data from pure and mixed stands of Scots pine (Pinus sylvestris $L$.) and European beech (Fagus sylvatica $L$.) through Europe
}

\author{
Michael Heym ${ }^{1}$ - Ricardo Ruíz-Peinado ${ }^{2,3}$ • Miren Del Río ${ }^{2,3}$ • Kamil Bielak ${ }^{4}$. \\ David I. Forrester ${ }^{5}$. Gerald Dirnberger ${ }^{6} \cdot$ Ignacio Barbeito $^{7} \cdot$ Gediminas Brazaitis $^{8}$. \\ Indrẻ Ruškytké $^{8}$ - Lluís Coll ${ }^{9}$ Marek Fabrika ${ }^{10}$ • Lars Drössler ${ }^{11}$ • Magnus Löf ${ }^{11}$. \\ Hubert Sterba $^{6}$ - Václav Hurt ${ }^{12}$ - Viktor Kurylyak ${ }^{13}$ - Fabio Lombardi ${ }^{14}$. \\ Dejan Stojanović ${ }^{15}$. Jan Den Ouden ${ }^{16} \cdot$ Renzo Motta $^{17}$ - Maciej Pach ${ }^{18}$. \\ Jerzy Skrzyszewski ${ }^{18}$ • Quentin Ponette ${ }^{19}$. Géraud De Streel ${ }^{19}$ • Vit Sramek ${ }^{20}$. \\ Tomáš Čihák ${ }^{21}$ - Tzvetan M. Zlatanov ${ }^{22}$ - Admir Avdagic ${ }^{23}$ • Christian Ammer ${ }^{24}$. \\ Kris Verheyen $^{25}$ • Buraczyk Włodzimierz ${ }^{4}$. Andrés Bravo-Oviedo ${ }^{2,3}$ • Hans Pretzsch ${ }^{1}$
}

Received: 24 January 2017 / Accepted: 25 July 2017 /Published online: 28 August 2017

(C) INRA and Springer-Verlag France SAS 2017

\begin{abstract}
- Key message This data set provides unique empirical data from triplets of Scots pine (Pinus sylvestris $\mathbf{L}$.) and European beech (Fagus sylvatica L.) across Europe. Dendrometric variables are provided for 32 triplets, 96 plots, 7555 trees and 4695 core samples. These data contribute to our understanding of mixed stand dynamics. Dataset access at http://dx.doi.org/10. 5061/dryad.8v04m. Associated metadata available at https://metadata-afs.nancy.inra.fr/geonetwork/apps/ georchestra/?uuid=b3e098ca-e681-4910-90990e25d3b4cd52\&hl=eng.
\end{abstract}

Handling Editor: Marianne Peiffer

Contribution of the co-authors

All co-authors contributed to the data set with establishing the triplets, supervising and undertaking field campaigns, data providing, synchronizing core data. M.H. wrote the data paper, all coauthors reviewed it and H.P. coordinated the transect study.

Electronic supplementary material The online version of this article (http://dx.doi.org/10.1007/s13595-017-0660-z) contains supplementary material, which is available to authorized users.

Michael Heym

Michael.Heym@1rz.tum.de

Ricardo Ruíz-Peinado

ruizpein@inia.es

Miren Del Río

delrio@inia.es
Keywords EuMIXFOR data $\cdot$ Mixed and monospecific stands $\cdot$ Mixed stand dynamics $\cdot$ Scots pine $\cdot$ European beech

\section{Background}

During the last decade, empirical research often showed superiority of ecosystem functions of mixed forest stands compared to monocultures, e.g. productivity (Río and Sterba 2009; Pretzsch et al. 2010, 2013; Vallet and Perot 2011, Condés et al. 2013; Bielak et al. 2014; Liang et al. 2016), structural diversity (Río et al. 2016a) or stability (Knoke et al. 2008). However, the underlying processes are often still poorly understood. Moreover, generalizations require empirical data from different growing conditions, e.g. soil and climate. This unique data set includes 32 triplets across 16 European countries (Fig. 1) representing different ecoregions. Each triplet includes two pure stands and one with the two species in mixture, all with similar climatic and soil conditions. Effects of mixing these species can be analysed with respect to their corresponding pure stands. Under the umbrella

\footnotetext{
Kamil Bielak

kamil.bielak@wl.sggw.pl

David I. Forrester

david.forrester@wsl.ch

Gerald Dirnberger

gerald.dirnberger@boku.ac.at
} 
of COST Action FP1206 EuMIXFOR (European Network on Mixed Forests), these triplets were established for the common European tree species, Scots pine and European beech. These species differ in their functional traits, and the data set provides a unique opportunity to develop a general understanding of the causes and patterns of mixing responses

\section{Methods}

\subsection{Study sites}

All stands (plots) represent mostly even-aged and monolayered forests. Intra-specific age (species in pure vs species in mixed stand) within each triplet is always similar; however, inter-specific age may differ. Plots within a triplet are mostly rectangular in shape ranging from 0.01 to 1.6 ha in size. The geographical location, altitude, slope, aspect, mean annual temperature, annual precipitation and substrate are available for each plot (Table 1). Due to the established gradient, these variables differ between triplets but are similar within each triplet.

Ignacio Barbeito
ignacio.barbeito@nancy.inra.
Gediminas Brazaitis
gediminas.brazaitis@asu.lt
Indre Ruškytkė
Indre.Ruskyte@asu.lt
Lluís Coll
lluis.coll@ctfc.es
Marek Fabrika
fabrika@tuzvo.sk
Lars Drössler
lars.drossler@slu.se
Magnus Löf
Magnus.Lof@slu.se
Hubert Sterba
hubert.sterba@boku.ac.at
Václav Hurt
vhurt@email.cz
Viktor Kurylyak
vikur@ukr.net
Fabio Lombardi
fabio.lombardi@unirc.it
Dejan Stojanović
dejan.stojanovic@uns.ac.rs
Jan Den Ouden
Jan.denOuden@wur.nl

\subsection{Survey protocol}

A standard protocol for data collection was established and applied for each triplet. For all trees exceeding diameter at breast height of $7 \mathrm{~cm}$, mandatory attributes (Table 2) were defined and collected including tree number $(\mathrm{Nr})$, tree species, diameter at breast height (dbh), tree height (h) and crown base height (cbh). In addition, on each plot, increment core samples for 10-20 dominant trees per species and angle count samples (acs) for the sampled trees were collected (Bitterlich 1952). Specific information is assigned to each tree indicating its status (alive, dead or damaged). Only standing trees were recorded. Spatial location (Cartesian coordinates) of individual trees was defined as an optional variable but was measured on most plots.

The tree numbering system is in ascending order starting from 1 for trees located within the plot and, if recorded, 901 for trees outside but with part of their crowns inside the plot. For all labelled trees, the corresponding species was determined. Diameter at breast height was measured for all trees using girth tape while a digital hypsometer was used for measuring tree height

\author{
Renzo Motta \\ renzo.motta@unito.it \\ Maciej Pach \\ rlpach@cyf-kr.edu.pl \\ Jerzy Skrzyszewski \\ rlskrzys@cyf-kr.edu.pl \\ Quentin Ponette \\ quentin.ponette@uclouvain.be \\ Géraud De Streel \\ geraud.destreel@uclouvain.be \\ Vit Sramek \\ sramek@vulhm.cz \\ Tomáš Čihák \\ cihak@vulhm.cz \\ Tzvetan M. Zlatanov \\ tmzlatanov@gmail.com \\ Admir Avdagic \\ a.avdagic@sfsa.unsa.ba \\ Christian Ammer \\ Christian.Ammer@forst.uni-goettingen.de \\ Kris Verheyen \\ Kris.Verheyen@UGent.be \\ Buraczyk Włodzimierz \\ wburaczyk@wp.pl \\ Andrés Bravo-Oviedo \\ bravo@inia.es
}


and crown base height. Increment cores aiming to reach the pith were taken at $1.30 \mathrm{~m}$ height in north and east directions from the stem using increment borers. Likewise, 1-2 angle count samples (acs) were recorded using a relascope with basal area factor 4 or $1 \mathrm{~m}^{2} \mathrm{ha}^{-1}$. All data were stored using predefined templates. In total, 7555 trees were measured and 4695 core samples were taken.

For 24 triplets, optional data of individual tree location and crown radii were collected. Crown radii (m) were mostly measured in N, NE, E, SE, S, SW, W and NW cardinal directions with a minimum of four directions. Tree spatial information is given in Cartesian coordinates referring to a point of origin, e.g. the south west corner post of the corresponding plot.

\subsection{Data processing — stand level data}

Stand characteristics such as mean tree dimension, basal area $\left(\mathrm{BA} \mathrm{m} \mathrm{m}^{2} \mathrm{ha}^{-1}\right)$ and volume stock per hectare $\left(\mathrm{V} \mathrm{m}^{3} \mathrm{ha}^{-1}\right)$ were derived for each plot and species group, pine and beech. Additional coniferous and deciduous spe-

Hans Pretzsch

Hans.Pretzsch@1rz.tum.de

1 Chair for Forest Growth and Yield Science, Technische Universität München, Munich, Germany

2 Department of Silviculture and Forest Systems Management, INIA-CIFOR, Madrid, Spain

3 Sustainable Forest Management Research Institute University of Valladolid \& INIA, Valladolid, Spain

4 Department of Silviculture, Warsaw University of Life Sciences, Warsaw, Poland

5 Swiss Federal Institute for Forest, Snow and Landscape Research WSL, Birmensdorf, Switzerland

6 Department of Forest and Soil Science, BOKU University of Natural Resources and Life Sciences, Vienna, Austria

7 Laboratoire d'Etude des Ressources Forêt Bois (LERFoB), INRA centre of Nancy, Champenoux, France

8 Institute of Forest Biology and Silviculture, Faculty of Forest Science and Ecology, Aleksandras Stulginiskis University, Studentu str. 11, Akademija, LT-53361 Kaunas dist, Lithuania

9 Department of Agriculture and Forest Engineering - Forest Sciences Centre of Catalonia (CTFC), University of Lleida, Lleida, Spain

10 Department of Forest Management and Geodesy, Faculty of Forestry, Technical University in Zvolen, Zvolen, Slovakia

11 Southern Swedish Forest Research Centre, Swedish University of Agricultural Sciences, Alnarp, Sweden

12 Department of Silviculture, Mendel University, Brno, Czech Republic cies within the mixed stands were grouped either as pine or beech, respectively. In pure stands, all additional species were assigned to the corresponding main species. Stand attributes are based on all surveyed living trees within the corresponding plot and are expressed per ha. A Petterson height curve function (Petterson 1955) was parameterized for each plot and species group. Missing tree heights and the height of quadratic mean diameter tree (hg) were calculated applying the corresponding height curve function. Stand volume was derived while taking into account each individual tree's diameter at breast height, derived tree height and species-specific form factors (Franz 1971). All stand characteristics (Table 3) were calculated using standard evaluation software available at the Chair of Forest Growth and Yield Science, TU München (Biber 2013).

\section{Access to data and metadata description}

The data set is available from the Dryad Digital Repository http://dx.doi.org/10.5061/dryad.8v04m (Heym

13 Forestry Academy of Sciences of Ukraine, Lviv, Ukraine

14 Dipartimento di AGRARIA, Università Mediterranea di Reggio Calabria, Contrada Melissari, Loc. Feo di Vito, 89122 Reggio Calabria, Italy

15 Institute of Lowland Forestry and Environment, University of Novi Sad, Novi Sad, Serbia

16 Forest Ecology and Forest Management Group, Wageningen University \& Research, Wageningen, The Netherlands

17 Department of Agricultural, Forest and Food Sciences DISAFA, University of Turin, Turin, Italy

18 Department of Silviculture, Institute of Forest Ecology and Silviculture, University of Agriculture, Krakow, Poland

19 Faculty of Bioscience Engineering \& Earth and Life Institute, Université catholique de Louvain, Louvain-la-Neuve, Belgium

20 Forestry and Game Management Research Institute, Opocno, Czech Republic

21 Forestry and Game Management Research Institute, Jíloviště, Czech Republic

22 Department of Silviculture, Forest Research Institute, Sofia, Bulgaria

23 Faculty of Forestry, University Sarajevo, Sarajevo, Bosnia and Herzegovina

24 Abteilung Waldbau und Waldökologie der gemäßigten Zonen, Georg-August-Universität Göttingen, Göttingen, Germany

25 Faculty of Bioscience Engineering, Forest \& Nature Lab, Ghent University, Melle, Gontrode, Belgium 
Fig. 1 Distribution of the triplet locations across Europe and distribution of European beech and Scots pine according EUFORGEN (www.euforgen. org). Triangles represent triplet locations

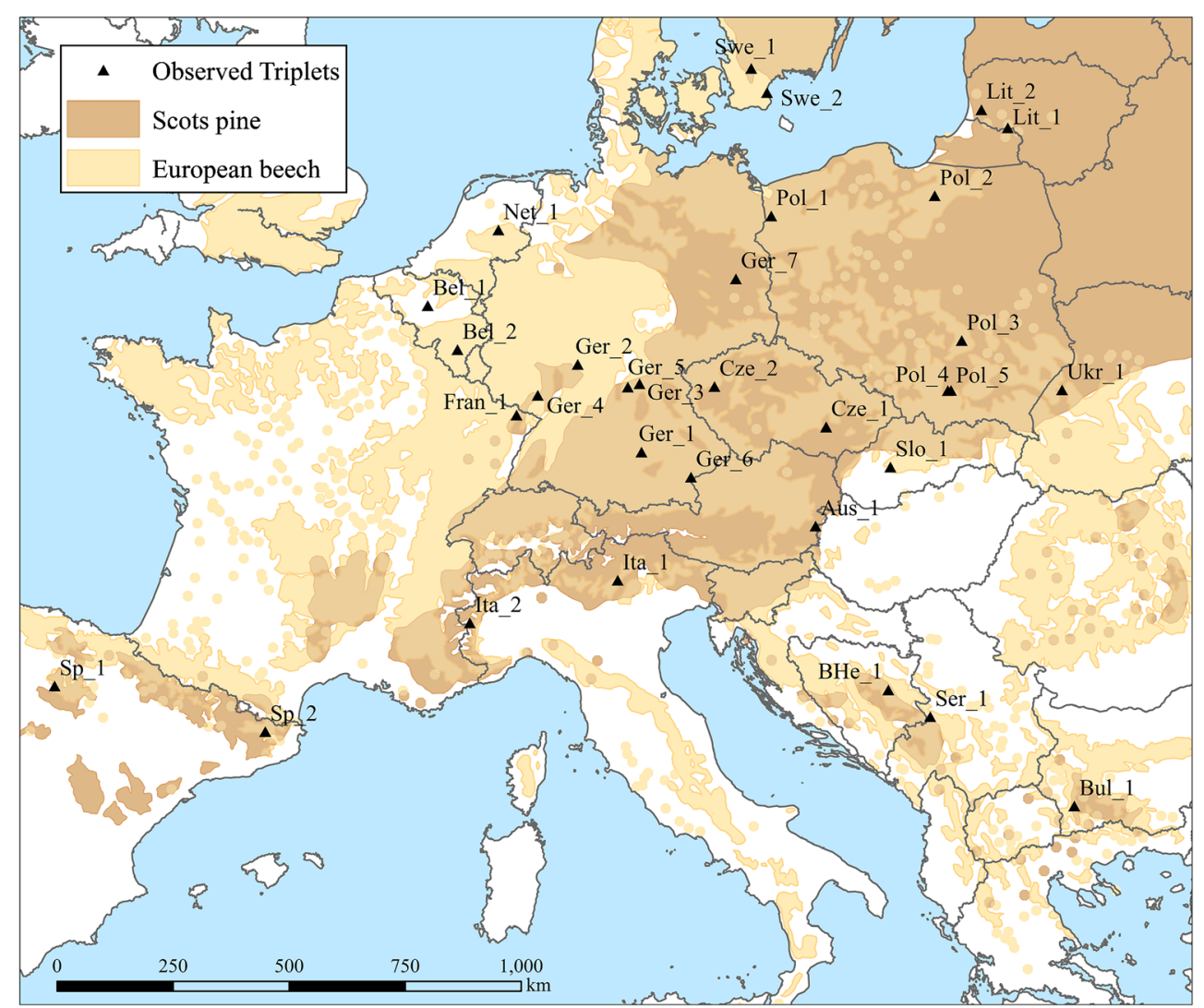

et al. 2017) and cover five files (Contact.txt, TripletInformation.txt, Trees.txt, Crowns.txt and Cores. txt). Contact.txt file includes all contact information of the specific data provider. Associated metadata available at https://metadata-afs.nancy.inra.fr/geonetwork/apps/ georchestra/?uuid=b3e098ca-e681-4910-9099$0 \mathrm{e} 25 \mathrm{~d} 3 \mathrm{~b} 4 \mathrm{~cd} 52 \& \mathrm{chl}=\mathrm{eng}$.

TripletInformation.txt provides plot and stand characteristics. The first two columns (Triplet and Plot) identify each plot. Plot characteristics cover year and month of the survey (year and month), plot size (area), location (longitude and latitude), elevation above sea level (altitude), inclination (slope), exposition (aspect), temperature $(t)$, precipitation $(p)$ and substrate (substrate). Stand characteristics cover tree species (species), age (Age), number of trees $(N)$, quadratic mean diameter $(d g)$ and corresponding height $(h g)$, basal area $(B A)$ and merchantable stand volume over bark $(V)$. All variables refer to 1 ha and the main species (species) pine or beech, respectively. Additional species are assigned either as pine or beech as described in methods.
Trees.txt includes all measured tree attributes. Each row describes one tree and can be identified by columns Triplet, Plot and Nr. Tree information covers scientific species name (species), tree's spatial information ( $x$ and $y$ ), diameter at breast height $(d b h)$, tree height $(h)$, crown base height $(c b h)$ and two angle count measurements for the cored trees (acs_l and acs_2). Column cored distinguishes cored trees (1) and non-cored trees (0). Any additional information is given in column remarks.

Crowns.txt contains all measured crown radii (distance) with its corresponding cardinal direction (azimuth). Column Triplet, Plot and $\mathrm{Nr}$ ensure the link to the corresponding tree in Trees.txt. In addition $y \_$axis_to_north describes the deviation of the $y$-axis to north direction.

Cores.txt file contains all year ring width values $(r w)$ for each year (year) and the two different directions (azimuth). Column Triplet, Plot and $\mathrm{Nr}$ ensure unique tree identification and link the corresponding tree attributes.

Missing information is always described using NA. All tables can be linked based on the columns Triplet, Plot and 
Table 1 Overview of all triplets with: Country; corresponding country, Triplet name; name of the triplet in Fig. 1, Triplet; triplet identification number, longitude and latitude (for mixed stands), age; average age for the triplet (years, yr), altitude; elevation above sea level (m), slope (degrees), aspect (degrees [clockwise from north]), $t$; mean annual temperature $\left(\mathrm{C}^{\circ}\right), p$; annual precipitation $(\mathrm{mm})$ and substrate. Altitude, slope and aspect represent average plot values

\begin{tabular}{|c|c|c|c|c|c|c|c|c|c|c|c|}
\hline Country & Triplet name & Triplet & Longitude & Latitude & Age & Altitude & Slope & Aspect & $t$ & $p$ & Substrate \\
\hline Austria & Aus_1 & 1048 & $16^{\circ} 23^{\prime} 20.00^{\prime \prime}$ & $47^{\circ} 22^{\prime} 34.00^{\prime \prime}$ & 40 & 525 & 18 & 213 & 8.5 & 750 & Loamy sand \\
\hline Belgium & $\mathrm{Bel} 2$ & 1063 & $04^{\circ} 19^{\prime} 29.60^{\prime \prime}$ & $50^{\circ} 45^{\prime} 06.10^{\prime \prime}$ & 115 & 160 & 1 & 270 & 7.5 & 852 & Loam \\
\hline Belgium & Bel_-1 & 1057 & $05^{\circ} 27^{\prime} 00.00^{\prime \prime}$ & $50^{\circ} 01^{\prime} 48.00^{\prime \prime}$ & 150 & 545 & 3 & 180 & 11 & 1175 & Stony loam \\
\hline $\begin{array}{l}\text { Bosnia \& } \\
\text { Herzegovina }\end{array}$ & BHe_1 & 1059 & $18^{\circ} 29^{\prime} 56.12^{\prime \prime}$ & $44^{\circ} 13^{\prime} 34.56^{\prime \prime}$ & 135 & 697 & 25 & 225 & 9.5 & 939 & $\begin{array}{c}\text { Humus-silicate } \\
\text { soil-ranker }\end{array}$ \\
\hline Bulgaria & Bul 1 & 1047 & $23^{\circ} 21^{\prime} 03.00^{\prime \prime}$ & $41^{\circ} 53^{\prime} 43.00^{\prime \prime}$ & 65 & 1187 & 18 & 350 & 6 & 750 & Loamy sand \\
\hline Czech Republic & Cze_1 & 1049 & $16^{\circ} 36^{\prime} 08.78^{\prime \prime}$ & $49^{\circ} 18^{\prime} 14.40^{\prime \prime}$ & 45 & 440 & 7 & 45 & 7.5 & 620 & Cambisol mezotrofic \\
\hline Czech Republic & Cze_2 & 1058 & $13^{\circ} 12^{\prime} 45.90^{\prime \prime}$ & $49^{\circ} 58^{\prime} 02.50^{\prime \prime}$ & 55 & 554 & 11 & 328 & 7.1 & 656 & $\begin{array}{l}\text { Dystric and podzol } \\
\text { cambisol }\end{array}$ \\
\hline France & Fra 1 & 1040 & $07^{\circ} 29^{\prime} 13.60^{\prime \prime}$ & $48^{\circ} 58^{\prime} 41.80^{\prime \prime}$ & 60 & 275 & 34 & 315 & 9.7 & 948 & Sandstone sandy soil \\
\hline Germany & Ger 1 & 1033 & $11^{\circ} 14^{\prime} 12.49^{\prime \prime}$ & $48^{\circ} 34^{\prime} 57.95^{\prime \prime}$ & 57 & 430 & 1 & 45 & 8.5 & 700 & Slightly loamy sand \\
\hline Germany & Ger_2 & 1031 & $09^{\circ} 03^{\prime} 54.36^{\prime \prime}$ & $50^{\circ} 06^{\prime} 48.74^{\prime \prime}$ & 55 & 250 & 0 & & 9 & 720 & Slightly loamy sand \\
\hline Germany & Ger 3 & 1032 & $10^{\circ} 58^{\prime} 13.12^{\prime \prime}$ & $49^{\circ} 53^{\prime} 11.64^{\prime \prime}$ & 47 & 250 & 2 & 30 & 8 & 650 & Loamy sand \\
\hline Germany & Ger 4 & 1071 & $08^{\circ} 01^{\prime} 03.88^{\prime \prime}$ & $49^{\circ} 24^{\prime} 57.77^{\prime \prime}$ & 65 & 40 & 1 & 60 & 9 & 675 & Loamy sand \\
\hline Germany & Ger_5 & 1034 & $08^{\circ} 10^{\prime} 48.58^{\prime \prime}$ & $48^{\circ} 59^{\prime} 11.66^{\prime \prime}$ & 57 & 370 & 3 & 0 & 10 & 675 & Slightly loamy sand \\
\hline Germany & Ger 6 & 1070 & $12^{\circ} 44^{\prime} 08.30^{\prime \prime}$ & $48^{\circ} 11^{\prime} 12.47^{\prime \prime}$ & 65 & 400 & 0 & & 8 & 560 & Slightly loamy sand \\
\hline Germany & Ger 7 & 1061 & $13^{\circ} 36^{\prime} 54.28^{\prime \prime}$ & $52^{\circ} 04^{\prime} 45.47^{\prime \prime}$ & 80 & 73 & 0 & & 8.6 & 520 & Sandy \\
\hline Italy & Ita $\overline{1}$ & 1055 & $10^{\circ} 56^{\prime} 10.61^{\prime \prime}$ & $46^{\circ} 04^{\prime} 02.93^{\prime \prime}$ & 40 & 1034 & 8 & 31 & 7.8 & 1050 & Cutanic luvisoil \\
\hline Italy & Ita 2 & 1062 & $07^{\circ} 03^{\prime} 53.30^{\prime \prime}$ & $44^{\circ} 54^{\prime} 12.49^{\prime \prime}$ & 55 & 1475 & 25 & 315 & 7.9 & 938 & Inceptisol \\
\hline Lithuania & Lit_1 & 1051 & $22^{\circ} 24^{\prime} 24.10^{\prime \prime}$ & $55^{\circ} 04^{\prime} 47.30^{\prime \prime}$ & 90 & 25 & 0 & & 6.5 & 750 & $\begin{array}{l}\text { Sand and slightly } \\
\text { loamy sand }\end{array}$ \\
\hline Lithuania & Lit_2 & 1052 & $21^{\circ} 32^{\prime} 23.44^{\prime \prime}$ & $55^{\circ} 27^{\prime} 02.80^{\prime \prime}$ & 111 & 20 & 0 & & 6.5 & 800 & $\begin{array}{l}\text { Sand and slightly } \\
\text { loamy sand }\end{array}$ \\
\hline Netherlands & Net_1 & 1043 & $06^{\circ} 01^{\prime} 20.42^{\prime \prime}$ & $52^{\circ} 25^{\prime} 40.55^{\prime \prime}$ & 47 & 34 & 2 & 68 & 9.7 & 825 & Coarse sand \\
\hline Poland & Pol_2 & 1036 & $19^{\circ} 54^{\prime} 42.27^{\prime \prime}$ & $53^{\circ} 48^{\prime} 19.15^{\prime \prime}$ & 81 & 136 & 0 & & 7.9 & 666 & Loamy sand and sand \\
\hline Poland & Pol 1 & 1035 & $14^{\circ} 36^{\prime} 17.51^{\prime \prime}$ & $53^{\circ} 20^{\prime} 07.40^{\prime \prime}$ & 55 & 60 & 0 & & 9.2 & 556 & Slightly loamy sand \\
\hline Poland & Pol_3 & 1037 & $20^{\circ} 41^{\prime} 08.90^{\prime \prime}$ & $50^{\circ} 59^{\prime} 27.96^{\prime \prime}$ & 76 & 383 & 2 & 275 & 7.8 & 662 & $\begin{array}{l}\text { Sandstone loamy } \\
\text { sand and loam }\end{array}$ \\
\hline Poland & Pol 4 & 1044 & $20^{\circ} 13^{\prime} 45.84^{\prime \prime}$ & $50^{\circ} 01^{\prime} 27.60^{\prime \prime}$ & 57 & 208 & 1 & 0 & 8.2 & 650 & Slightly loamy sand \\
\hline Poland & Pol 5 & 1045 & $20^{\circ} 19^{\prime} 37.26^{\prime \prime}$ & $50^{\circ} 01^{\prime} 36.00^{\prime \prime}$ & 55 & 213 & 0 & & 8.2 & 650 & Loamy sand \\
\hline Serbia & Ser 1 & 1056 & $19^{\circ} 37^{\prime} 30.00^{\prime \prime}$ & $43^{\circ} 42^{\prime} 17.40^{\prime \prime}$ & 75 & 1080 & 21 & 0 & 7.7 & 1020 & Loam with a little sand \\
\hline Slovakia & Slo_1 & 1046 & $18^{\circ} 31^{\prime} 11.19^{\prime \prime}$ & $48^{\circ} 33^{\prime} 09.18^{\prime \prime}$ & 55 & 524 & 15 & 90 & 6.9 & 730 & Cambisoil \\
\hline Spain & $\mathrm{Sp} \overline{1}$ & 1042 & $03^{\circ} 10^{\prime} 19.00^{\prime \prime} \mathrm{W}$ & $42^{\circ} 05^{\prime} 57.00^{\prime \prime}$ & 40 & 1293 & 47 & 120 & 8.9 & 860 & Sandy loam \\
\hline Spain & Sp_2 & 1041 & $02^{\circ} 15^{\prime} 44.23^{\prime \prime}$ & $42^{\circ} 10^{\prime} 18.09^{\prime \prime}$ & 50 & 1116 & 30 & 135 & 8 & 1100 & Loam slightly clay \\
\hline Sweden & Swe_1 & 1054 & $13^{\circ} 35^{\prime} 35.00^{\prime \prime}$ & $56^{\circ} 09^{\prime} 12.00^{\prime \prime}$ & 80 & 120 & 13 & 222 & 8 & 700 & Loamy sand \\
\hline Sweden & Swe 2 & 1053 & $14^{\circ} 11^{\prime} 46.00^{\prime \prime}$ & $55^{\circ} 42^{\prime} 33.00^{\prime \prime}$ & 65 & 25 & 8 & 120 & 7 & 800 & Sandy till \\
\hline Ukraine & $\mathrm{Ukr}_{-} 1$ & 1060 & $23^{\circ} 39^{\prime} 44.00^{\prime \prime}$ & $49^{\circ} 57^{\prime} 05.00^{\prime \prime}$ & 105 & 316 & 0 & & 7.6 & 673 & Slightly loamy sand \\
\hline
\end{tabular}

$N r$. Detailed description of the metadata can be found in the supplementary material (EuMIXFOR Scots pine - European beech data.xlsx).

\section{Technical validation}

Validation of each data set was performed including crosschecks by hand, graphical and numerical tests. First, the unit of tree attributes and year ring width was carefully validated. Tree and corresponding core labels were compared manually and corrected where needed. Cross-dating of radial increment was performed by each collaborator and inconsistent cores were dropped. The relationship of individual tree height to its corresponding diameter at breast height, expressed by a Petterson height curve, was analysed for each triplet, shown as an example for triplet 1032 in Fig. 2.

Crown base height was validated by visualizing cbh and corresponding tree height. If spatial information was
Table 2 Overview of measured mandatory and optional descriptive and dendrometric attributes

\begin{tabular}{|c|c|c|}
\hline & Variable & Description \\
\hline \multirow[t]{15}{*}{ Mandatory } & Longitude & Plot specific \\
\hline & Latitude & Plot specific \\
\hline & Altitude & Plot specific; $m$ (E. a.s.1.) \\
\hline & Slope & Plot specific; degrees \\
\hline & Aspect & Plot specific; degrees \\
\hline & Plot size & Plot specific; hectares \\
\hline & $\begin{array}{l}\text { Date of } \\
\quad \text { establishment/measurement }\end{array}$ & Triplet specific; yyyy-mm \\
\hline & Age & $\begin{array}{l}\text { Species and plot specific; } \\
\text { years }\end{array}$ \\
\hline & Tree number & $\begin{array}{l}\text { Living trees; ascending } \\
\text { order }\end{array}$ \\
\hline & Tree species & Scientific species name \\
\hline & Diameter at breast height & Tree specific; $\mathrm{cm}$ \\
\hline & Tree height & Tree specific; m \\
\hline & Crown base height & Tree specific; $m$ \\
\hline & Increment cores & $\begin{array}{l}2 \text { core samples/tree, } 10-20 \\
\text { trees/plot/species }\end{array}$ \\
\hline & Local density & $\begin{array}{l}\text { 1-2 angle count measurements/cored } \\
\text { tree; } \mathrm{m}^{2} \mathrm{ha}^{-1}\end{array}$ \\
\hline \multirow[t]{3}{*}{ Optional } & $x$-coordinate & Tree specific (Cartesian) \\
\hline & $y$-coordinate & Tree specific (Cartesian) \\
\hline & Crown radii & $\begin{array}{l}\text { Tree specific; azimuth } \\
\text { (degrees) and distance } \\
\text { (m) }\end{array}$ \\
\hline
\end{tabular}


Table 3 Example (triplet 1033) of main stand characteristics per plot with: Country; corresponding country for the triplet, Triplet; triplet identification number, Plot plot identification (pibe; mixed stand pi; pure pine, be; pure beech), Year; year of survey, Species; species name,
Age; plot age at survey, $N$; number of trees, $d g$; quadratic mean diameter $(\mathrm{cm}), h g$ height of quadratic mean diameter $(\mathrm{m}), B A$; basal area $\left(\mathrm{m}^{2}\right)$ and $V$; merchantable volume per hectare $\left(\mathrm{m}^{3}\right)$. All variables refer to the main species and 1 ha

\begin{tabular}{|c|c|c|c|c|c|c|c|c|c|c|}
\hline Country & Triplet & Plot & Year & Species & Age & $N$ & $\begin{array}{l}\mathrm{dg} \\
(\mathrm{cm})\end{array}$ & $\begin{array}{l}\mathrm{hg} \\
(\mathrm{m})\end{array}$ & $\begin{array}{l}\text { BA } \\
\left(\mathrm{m}^{2} \mathrm{ha}^{-1}\right)\end{array}$ & $\begin{array}{l}\text { V } \\
\left(\mathrm{m}^{3} \mathrm{ha}^{-1}\right)\end{array}$ \\
\hline \multirow[t]{5}{*}{ Germany } & \multirow[t]{5}{*}{1033} & pibe & 2013 & Pine & 50 & 330 & 25.5 & 22.8 & 16.8 & 175 \\
\hline & & pibe & 2013 & Beech & 50 & 818 & 15.7 & 19.5 & 15.9 & 157 \\
\hline & & pibe & 2013 & Total & & 1148 & & & 32.8 & 332 \\
\hline & & pi & 2013 & Pine & 65 & 286 & 33.2 & 26 & 24.7 & 295 \\
\hline & & be & 2013 & Beech & 53 & 1032 & 17 & 24 & 23.3 & 279 \\
\hline
\end{tabular}

available, visual inspection was applied to validate tree position and crown measurements. In addition, allometric relationships between diameter at breast height and crown projection area (cpa), calculated based on mean squared crown radii, $\mathrm{cpa}=a^{*} \mathrm{dbh}^{b}$, were validated visually (Fig. 3).

Location of border trees was inspected by means of graphical output. Core data were also compared with speciesspecific variation of basal area increment in pure and mixed stands within each triplet (Fig. 4).

All data were imported into an access database and technical plausibility checks were applied, e.g. data types or duplicates.

\section{Reuse potential and limits}

The original data set covers attributes at the tree level, increments cores, stand and triplet characteristics and has been used for different purposes by Pretzsch et al. (2015, 2016), Río et al. (2016b, 2017), Dirnberger et al.

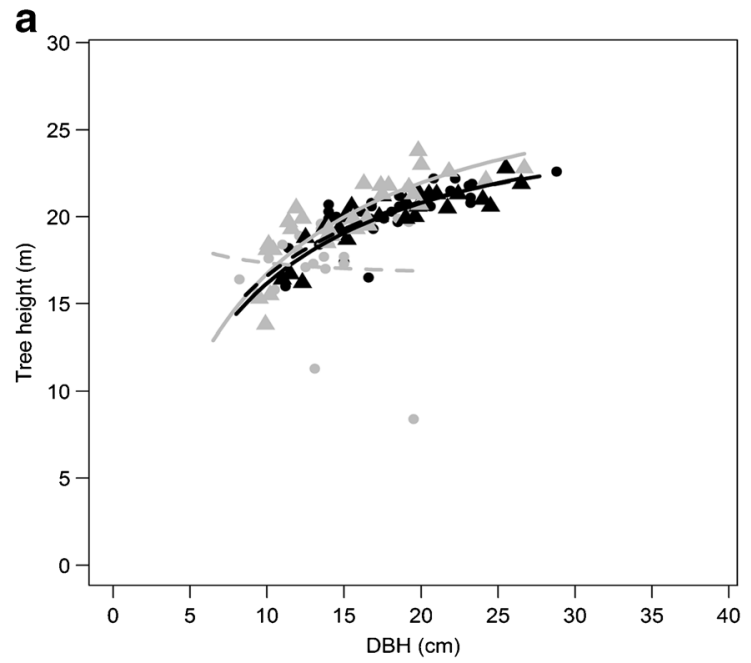

Fig. 2 Detecting (left side, a) and correcting (right side, b) inconsistent height measurements. Data from triplet 1032 for pine (black) and beech (grey) in pure (triangles) and mixed stands (circles). Dashed and solid
(2016) and Forrester et al. (2017). The data set presented here describes the original data, but ongoing projects can complement the published data by additional measurements of resource supply, nutritional status and wood properties. Furthermore, it has the potential to reveal the effect of site conditions on the mixing responses at the stand, species and individual tree level. Available spatial information allows analyses of crown projection (Dirnberger et al. 2016), crown architecture or light regimes. The available core data allow retrospective analyses and can be linked to corresponding tree attributes, e.g. Pretzsch et al. (2016) or Río et al. (2017). Reconstructing stand characteristics provides temporal stand level information, e.g. Pretzsch et al. (2015). Moreover, spatial information of most of the plots allows for distance dependent analysis at small scales.

However, for specific analyses, some plot sizes could be too small. Tree and stand characteristics are only available for the year of survey. Therefore, temporal analyses require reconstructions at the tree and stand levels.

b

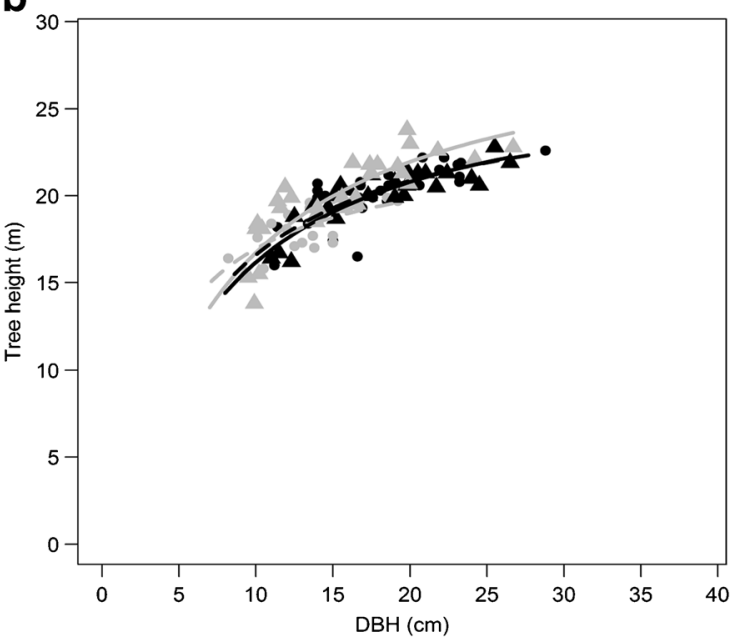

lines representing species-specific Petterson height curve for mixed and pure stands, respectively 
a

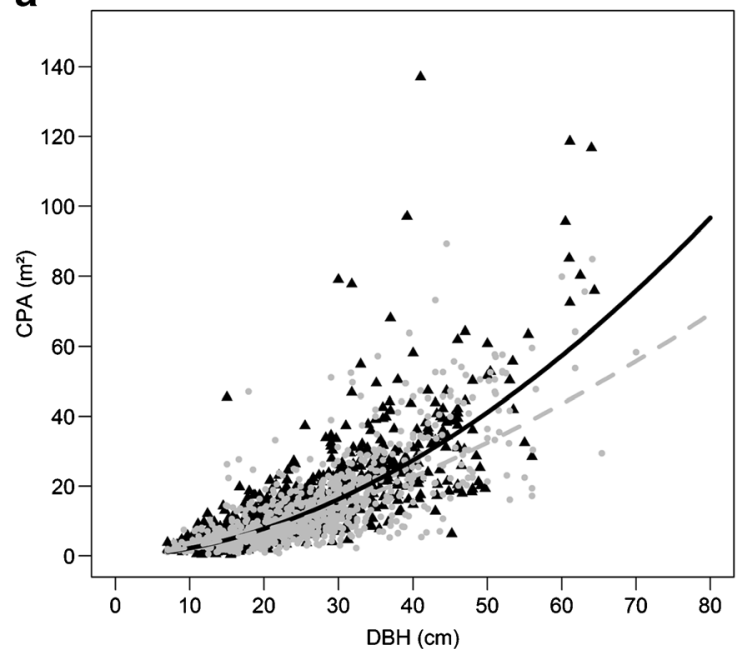

b

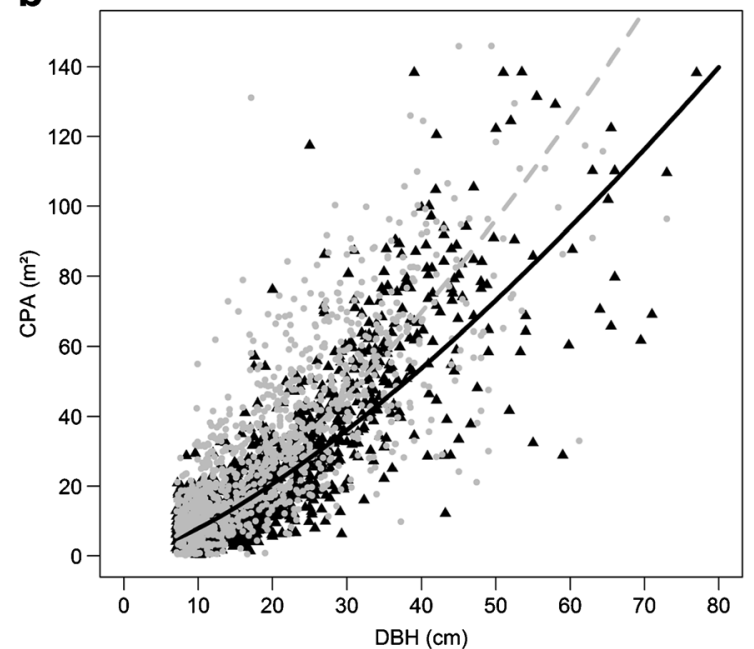

Fig. 3 Crown projection area (cpa) and diameter at breast height (dbh) for all measured pine (left side) and beech (right side) in pure (triangles) and mixed stands (circles). Allometric relationships are described by solid (pure stands) and dashed lines (mixed stands)

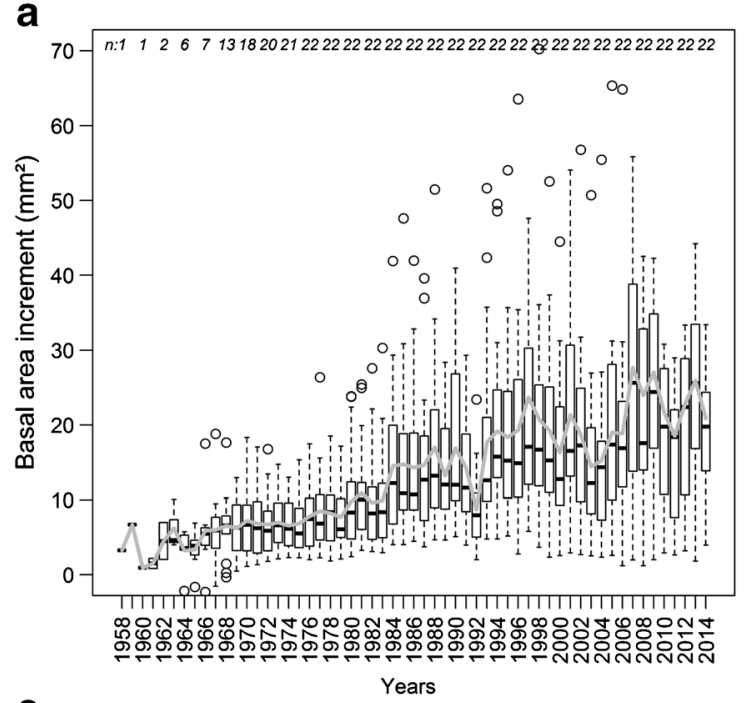

C

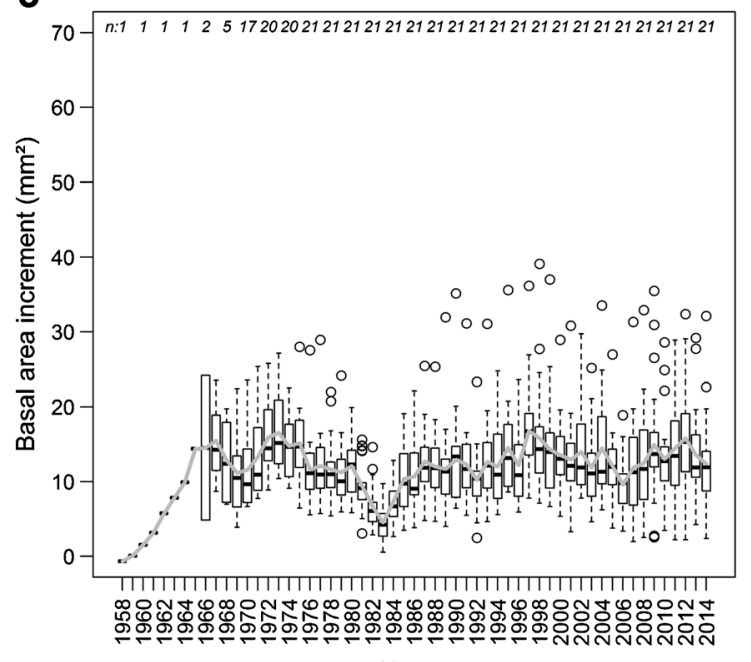

b

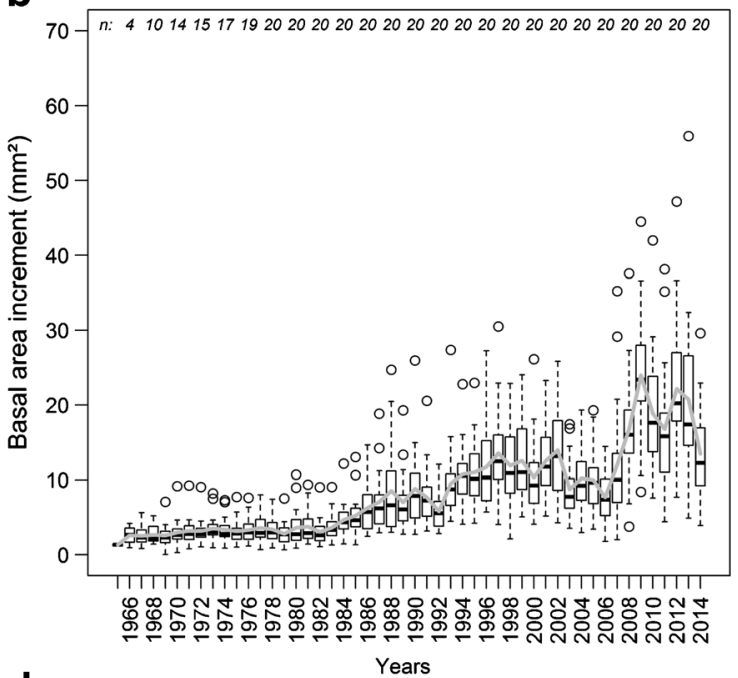

d

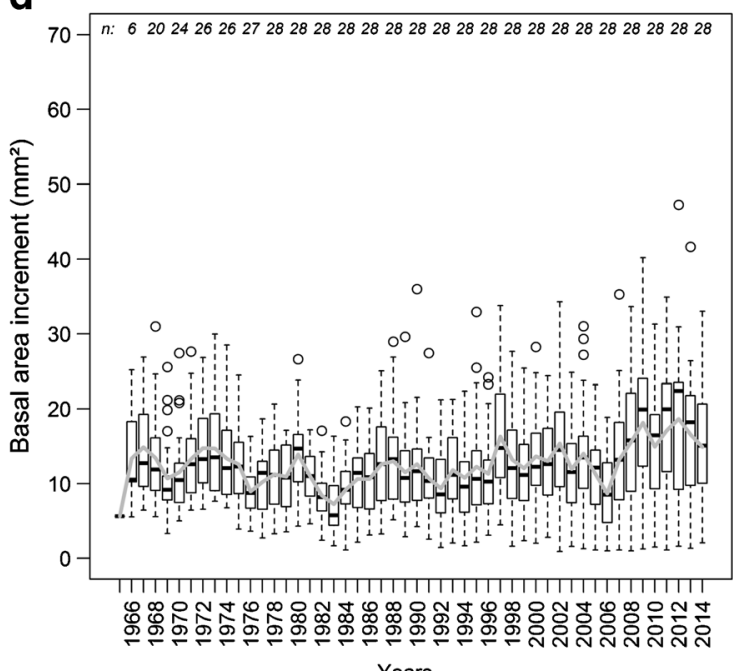

Fig. 4 Example of basal area increment per calendar year for beech (upper; $\mathbf{a}, \mathbf{b})$, and pine (lower; $\mathbf{c}, \mathbf{d})$, in pure (left; $\mathbf{a}, \mathbf{c})$ and mixed stand (right; $\mathbf{b}, \mathbf{d})$, for triplet 1035. Grey solid lines represent arithmetic means and $n$ refers to number of cores for years shown on $x$-axis 
Acknowledgements This article is based upon work from COST Action FP1206 (EuMIXFOR), supported by COST (European Cooperation in Science and Technology). All contributors thank their national funding institutions and the woodland owners for agreeing to establish, measure, analyse and reuse data from the triplets. Many thanks to the anonymous reviewer and data paper handling editor Marianne Peiffer for their helpful comments to improve the early draft of the manuscript.

\section{Compliance with ethical standards}

Funding Networking for the design and discussion of the transect study was supported by COST Association during FP1206 COST Action (EuMIXFOR: European mixed forests - Integration Scientific Knowledge in Sustainable Forest Management). Funding for the establishment of the plots and collection of the data belonged to co-author.

\section{References}

Biber P (2013) Kontinuität durch Flexibilität - Standardisierte Datenauswertung im Rahmen eines waldwachstumskundlichen Informationssystems, Allg. Forst- u. J.-Ztg., 184. Jg., 7/8:167-177

Bielak K, Dudzińska M, Pretzsch H (2014) Mixed stands of Scots pine (Pinus sylvestris L.) and Norway spruce (Picea abies (L.) Karst) can be more productive than monocultures. Evidence from over 100 years of observation of long-term experiments. For Syst 23:573-589

Bitterlich W (1952) Die Winkelzählprobe. Forstwiss Cbl 71:215-225

Condés S, Río M, Sterba H (2013) Mixing effect on volume growth of Fagus sylvatica and Pinus sylvestris is modulated by stand density. For Ecol Manag 292:86-95

Dirnberger G, Sterba H, Condés S, Ammer C, Annighöfer P, Avdagić A, Bielak K, Brazaitis G, Coll L, Heym M, Hurt V, Kurylyak V, Motta R, Pach M, Ponette Q, Ruiz-Peinado R, Skrzyszewski J, Šrámek V, de Streel G, Svoboda M, Zlatanov T, Pretzsch H (2016) Species proportions by area in mixtures of Scots pine (Pinus sylvestris L.) and European beech (Fagus sylvatica L.) Eur J For Res. http://dx. doi.org/10.1007/s10342-016-1017-0

Forrester DI, Ammer C, Annighöfer PJ, Barbeito I, Bielak K, BravoOviedo A, Coll L, Río Md, Drössler L, Heym M, Hurt V, Löf M, Ouden Jd, Pach M, Pereira MG, Plaga B, Ponette Q, Skrzyszewski J, Sterba H, Svoboda M, Zlatanov T, Pretzsch H (2017) Effects of crown architecture and stand structure on light absorption in mixed and monospecific Fagus sylvatica and Pinus sylvestris forests along a productivity and climate gradient through Europe. J Ecol 00:1-15. http://dx.doi.org/10.1111/1365-2745.12803

Franz F (1971) Funktionen und Tabellen der Derbholzformhöhen für die wichtigsten Baumarten in Bayern. München, Manuskriptdruck, Lehrstuhl für Waldwachstumskunde, Technische Universität München, unpublished

Heym M, Ruíz-Peinado R, del Río M, Bielak K, Forrester DI, Dirnberger G, Barbeito I, Brazaitis G, Ruškyte I, Coll L, Fabrika M, Drössler L, Löf M, Sterba H, Hurt V, Kurylyak V, Lombardi F, Stojanović D, den Ouden J, Motta R, Pach M, Skrzyszewski J, Ponette Q, de Streel G, Sramek V, Čihák T, Zlatanov TM, Avdagic A, Ammer C, Verheyen K, Włodzimierz B, Bravo-Oviedo A, Pretzsch H (2017) EuMIXFOR empirical forest mensuration and ring width data from pure and mixed stands of Scots pine (Pinus sylvestris L.) and European beech (Fagus sylvatica L.) through Europe. Dryad Digital Repository. [Dataset]. http://dx.doi.org/10.5061/dryad. $8 \mathrm{v} 04 \mathrm{~m}$

Knoke T, Ammer H, Stimm B, Mosandl R (2008) Admixing broadleaved to coniferous tree species: a review on yield, ecological stability and economics. Eur J For Res 127:89-101
Liang J, Crowther TW, Picard N, Wiser S, Zhou M, Alberti G, Schulze ED, McGuire AD, Bozzato F, Pretzsch H, de-Miguel S, Paquette A. Hérault B, Scherer-Lorenzen M, Barrett CB, Glick HB, Hengeveld GM, Nabuurs G-J, Pfautsch S, Viana H, Vibrans AC, Ammer C, Schall P, Verbyla D, Tchebakova N, Fischer M, Watson JV, HYH C, Lei X, Schelhaas M-J, Lu H, Gianelle D, Parfenova EI, Salas C, Lee E, Lee B, Seok Kim H, Bruelheide H, Coomes DA, Piotto D, Sunderland T, Schmid B, Gourlet-Fleury S, Sonké B, Tavani R, Zhu J, Brandl S, Vayreda J, Kitahara F, Searle EB, Neldner VJ, Ngugi MR, Baraloto C, Frizzera L, Bałazy R, Oleksyn J, ZawiłaNiedźwiecki T, Bouriaud O, Bussotti F, Finér L, Jaroszewicz B, Jucker T, Valladares F, Jagodzinski AM, Peri PL, Gonmadje C, Marthy W, O'Brien T, Martin EH, Marshall AR, Rovero F, Bitariho R, Niklaus PA, Alvarez-Loayza P, Chamuya N, Valencia R, Mortier F, Wortel V, Engone-Obiang NL, Ferreira LV, Odeke DE, Vasquez RM, Lewis SL, Reich PB (2016) Positive biodiversityproductivity relationship predominant in global forests. Science 354(6309):196. http://dx.doi.org/10.1126/science.aaf8957

Petterson H (1955) Die Massenproduktion des Nadelwaldes. Mitt Forstlichen Forschungsanstalten Schwedens 45(1):1-392

Pretzsch H, Block J, Dieler J, Dong PH, Kohnle U, Nagel J, Spellmann H, Zingg A (2010) Comparison between the productivity of pure and mixed stands of Norway spruce and European beech along an ecological gradient. Ann For Sci 67:1-12

Pretzsch H, Bielak K, Block J, Bruchwald A, Dieler J, Ehrhart H-P, Kohnle U, Nagel J, Spellmann H, Zasada M, Zingg A (2013) Productivity of pure versus mixed stands of oak (Quercus petraea (MATT.) LIEBL. and Quercus robur L.) and European beech (Fagus Sylvatica L.) along an ecological gradient. Eur J For Res 132:263280

Pretzsch H, Río M, Ammer C, Avdagic A, Barbeito I, Bielak K, Brazaitis G, Coll L, Dirnberger G, Drössler L, Fabrika M, Forrester D, Heym M, Hurt V, Kurylyak V, Löf M, Lombardi F, Mohren F, Motta R, den Ouden J, Pach M, Ponette Q, Schütze G, Schweig J, Skrzyszewski J, Sramek V, Sterba H, Stojanović D, Svoboda M, Vanhellemont M, Verheyen K, Wellhausen K, Zlatanov T, Bravo-Oviedo A (2015) Growth and yield of mixed versus pure stands of Scots pine (Pinus sylvestris L.) and European beech (Fagus sylvatica L.) analysed along a productivity gradient through Europe. For Ecol Manag 373:149-166

Pretzsch H, Rio M, Schütze G, Ammer C, Annighöfer P, Avdagic A, Barbeito I, Bielak K, Brazaitis G, Coll L, Drössler L, Fabrika M, Forrester DI, Kurylyak V, Löf M, Lombardi F, Matovic B, Mohren F, Motta R, den Ouden J, Pach M, Ponette Q, Skzyszewski J, Sramek V, Sterba H, Svoboda M, Verheyen K, Zlatanov T, BravoOviedo A (2016) Mixing of Scots pine (Pinus sylvestris L.) and European beech (Fagus sylvatica L.) enhances structural heterogeneity, and the effect increases with water availability. For Ecol Manag 373:149-166

Río M, Sterba H (2009) Comparing volume growth in pure and mixed stands of Pinus sylvestris and Quercus pyrenaica. Ann For Sci 66:111

Río M, Pretzsch H, Alberdi I, Bielak K, Bravo F, Brunner A, Condés S, Ducey MJ, Fonseca T, von Lüpke N, Pach M, Peric S, Perot T, Souidi Z, Spathelf P, Sterba H, Tijardovic M, Tomé M, Vallet P, Bravo-Oviedo A (2016a) Characterization of the structure, dynamics, and productivity of mixed species stands: review and perspectives. Eur J For Res 135:23-49

Río M, Pretzsch H, Ruíz-Peinado R, Ampoorter E, Annighöfer P, Barbeito I, Bielak K, Brazaitis G, Coll L, Drössler L, Fabrika M, Forrester DI, Heym M, Hurt V, Kurylyak V, Löf M, Lombardi F, Madrickiene E, Matović B, Mohren F, Motta R, den Ouden J, Pach M, Ponette Q, Schütze G, Skrzyszewski J, Sramek V, Sterba H, Stojanović D, Svoboda M, Zlatanov T, Bravo-Oviedo A (2016b) Data from: Species interactions increase the temporal stability of community productivity in Pinus sylvestris-Fagus sylvatica 
mixtures across Europe. Dryad Digital Repository. [Dataset]. http:// dx.doi.org/10.5061/dryad.fq4tk

Río M, Pretzsch H, Ruíz-PeinadoR AE, Annighöfer P, Barbeito I, Bielak K, Brazaitis G, Coll L, Drössler L, Fabrika M, Forrester DI, Heym M, Hurt V, Kurylyak V, Löf M, Lombardi F, Madrickiene E, Matović B, Mohren F, Motta R, den Ouden J, Pach M, Ponette Q, Schütze G, Skrzyszewski J, Sramek V, Sterba H, Stojanović D,
Svoboda M, Zlatanov T, Bravo-Oviedo A (2017) Species interactions increase the temporal stability of community productivity in Pinus sylvestris-Fagus sylvatica mixtures across Europe. J Ecol 105(40):1032-1043. http://dx.doi.org/10.1111/1365-2745.12727

Vallet P, Perot T (2011) Silver fir stand productivity is enhanced when mixed with Norway spruce: evidence based on large-scale inventory data and a generic modelling approach. J Veg Sci 22:932-942 